\title{
Panorama das neoplasias uterinas no período de 2007 a 2016 no estado de Alagoas - Brasil
}

\author{
Overview of uterine neoplasms in the period 2007 to 2016 in the state of Alagoas - Brazil
}

\author{
Jailton Rocha Misael, Claudio José dos Santos Júnior, Ilma Falcão Silva, \\ Lídia Nathaly Gomes dos Santos, Fernando Batista Chicuta da Rocha, \\ Marcicléa Macedo de Lima Batista, Amanda Maria Monteiro Ferreira
}

Misael JR, Santos Júnior CJ, Batista MML, Silva IF, Santos LNG, Silva FBC, Ferreira AMM. Panorama das neoplasias uterinas no período de 2007 a 2016 no estado de Alagoas - Brasil / Overview of uterine neoplasms in the period 2007 to 2016 in the state of Alagoas - Brazil. Rev Med (São Paulo). 2019 nov.-dez.;98(6):396-402.

RESUMO: Objetivo: Analisar a variação temporal das neoplasias uterinas na população feminina do estado de Alagoas. Método: Estudo quantitativo, transversal e retrospectivo. Os dados utilizados referem-se a internações e óbitos por neoplasias uterinas em Alagoas, no período de 2007 a 2016, disponíveis nos Sistemas de Informação de Saúde e analisados por estatística descritiva de frequências absoluta e relativa. Resultados: Em Alagoas, de 2007 a 2016, identificou-se 3.525 mulheres diagnosticadas com neoplasias de útero, com média anual de 352,5 casos novos $( \pm$ 64,3 ), que foram responsáveis por 4.540 registros de internações hospitalares nas instituições assistenciais de saúde estadual, cuja média foi de 454 internações/ano $( \pm 77,2)$, e 1.391 óbitos, com média de 139 óbitos anuais $( \pm 13,99)$. Verificou-se que a população adulta apresentou maior número de óbitos, com 822 registros $(57,97 \%)$. Notou-se também elevada prevalência no público idoso, com 594 óbitos (41,89\%). Quanto à procedência, constatou-se que a $1^{\mathrm{a}}$ e a $7^{\mathrm{a}}$ microrregiões de saúde concentram $58,51 \%$ dos óbitos locais (830 registros). Conclusão: Em Alagoas, observa-se a necessidade de políticas pública de saúde da mulher efetivas, que aperfeiçoem o acesso precoce para o diagnóstico e o tratamento do câncer de colo uterino, visando à redução dos índices de morbimortalidade.

Descritores: Neoplasias uterinas/epidemiologia; Neoplasias uterinas/diagnóstico; Saúde da mulher; Saúde pública; Indicadores de morbimortalidade; Brasil/epidemiologia.

\begin{abstract}
Objective: To analyze the temporal variation of uterine neoplasms in the female population of the State of Alagoas. Method: Quantitative, cross-sectional and retrospective study. The data used refer to admissions and deaths from uterine neoplasms in Alagoas, from 2007 to 2016, available in the Health Information Systems and analyzed by descriptive statistics of absolute and relative frequencies. Results: In Alagoas from 2007 to 2016, 3,525 women diagnosed with uterine neoplasms were identified, with an annual mean of 352.5 new cases $( \pm 64.3)$, which were responsible for 4,540 records of hospital admissions in state health care institutions, with a mean of 454 admissions / year $( \pm 77.2)$, and 1,391 deaths, with a mean of 139 annual deaths $( \pm 13.99)$. It was verified that the adult population had a higher number of deaths, with 822 records $(57.97 \%)$. There was also a high prevalence among the elderly population, with 594 deaths $(41.89 \%)$. Regarding the provenance, it was verified that the $1 \mathrm{st}$ and 7 th micro-regions of health concentrate $58.51 \%$ of the local deaths ( 830 records). Conclusion: In Alagoas, there is a need for effective public health policies, that improve early access for the diagnosis and treatment of cervical cancer, aiming at reducing morbidity and mortality rates.
\end{abstract}

Keywords: Uterine neoplasms/epidemiology; Uterine neoplasias/ diagnosis; Women's health; Public health; Indicators of morbidity and mortality; Brazil/epidemiology.

Universidade Estadual de Ciências da Saúde de Alagoas - UNCISAL, Maceió, AL, Brasil.

ORCID: Misael JR - https://orcid.org/0000-0002-8433-7078; Santos Júnior CJ - https://orcid.org/0000-0002-2853-1968; Batista MML - https://orcid.org/0000-0001-6827-2617; Silva IF - https://orcid.org/0000-0002-2372-0444; Santos LNG - https://orcid.org/00000003-2896-8198; Silva FBC - https://orcid.org/0000-0002-0072-3351; Ferreira AMM - https://orcid.org/0000-0002-9357-4219. Email: jailton.enf@gmail.com; claudiosantos_al@hotmail.com; marciclea@yahoo.com.br; ilmafalcao1@gmail.com; lidia.nath@gmail.com; fernandochicuta@hotmail.com; amandamonteiroferreira@hotmail.com.

Endereço para correspondência: Jailton Rocha Misael. Rua Dr. Jorge de Lima, 114 - Trapiche da Barra. Maceió - AL. CEP: 57010300. E-mail: jailton.enf@gmail.com. 


\section{INTRODUÇÃO}

Como consequência da elevação da expectativa de vida, observa-se nas últimas décadas um processo de transformações demográficas na população brasileira $^{1}$, caracterizada pela marcante transição epidemiológica, sobretudo no que tange às doenças crônicas. Neste contexto, destacam-se as neoplasias malignas na população feminina, principalmente nos distintos segmentos uterinos que acometem prevalentemente mulheres de todo o mundo, em especial, países subdesenvolvidos e de baixa renda ${ }^{2}$.

A partir da década de 1980, a introdução do rastreamento do câncer de colo do útero na Atenção Primária à Saúde no Brasil consolida a adoção de um importante mecanismo de prevenção, e com a descentralização do rastreamento para a Estratégia de Saúde da Família permitiu o eficiente enfrentamento dos índices relacionados à morbimortalidade das neoplasias uterinas. No território brasileiro ainda há limitação de acesso e oferta aos distintos recursos especificos ${ }^{3}$, conferindo a persistência dos elevados e distintos índices vinculados ao câncer de útero no cenário nacional.

Embora apresente o maior potencial de prevenção e cura, o câncer do colo do útero ainda se configura como um eminente problema de saúde coletiva no Brasil, pois é responsável pela terceira maior incidência na população feminina brasileira, com exceção dos registros de câncer de pele não melanoma, e pelo maior número de óbitos de mulheres de 15 a 44 anos de idade ${ }^{4}$. Desse modo, as políticas públicas que envolvem os distintos níveis de prevenção são prioridades da agenda de saúde nacional e são inseridas no Plano de Ações Estratégicas para o Enfrentamento das Doenças Crônicas Não Transmissíveis no Brasil, implementadas pelo Ministério da Saúde, em $2011^{5}$.

Diante dos esforços do Ministério da Saúde no enfrentamento do câncer uterino, principalmente por meio das Diretrizes para o Rastreamento do Câncer do Colo do Útero, do ano de 2016, permite-se concentrar o rastreamento da população feminina através da realização do exame citopatológico da cérvice uterina, sobretudo em mulheres assintomáticas e sexualmente ativas, com idade entre 25 e 64 anos, anualmente e a cada três anos, após dois exames anuais consecutivos normais ${ }^{6}$.

Reconhece-se que coexistem distintos fatores que incidem diretamente na susceptibilidade do público feminino às neoplasias uterinas, a saber: desqualificação dos recursos humanos na saúde, fragilidade na implementação de acompanhamento integral institucionalizado, bem como na limitada participação ativa na promoção da saúde, associada à baixa escolaridade e ao limitado acesso aos serviços essenciais de saúde 7 . Como reflexo conjuntural, para o ano de 2018, estima-se o número de 22.970 casos novos de câncer de útero no Brasil, com maior concentração nas regiões Sudeste e Nordeste do país, com 7.820 e 7.500 diagnósticos, respectivamente . $^{6}$.

Não obstante, também para o ano de 2018, no estado de Alagoas há a estimativa de 390 novos casos de neoplasias uterinas, dos quais, 320 mulheres com o diagnóstico de câncer do colo de útero e 70 registros de câncer de corpo uterino ${ }^{6}$. Face ao exposto, reconhece-se a relevância de estudar o comportamento temporal do câncer uterino, pois permite analisar a efetividade das políticas pública de saúde local, principalmente no que envolve a prevenção das neoplasias de útero nos distintos serviços municipais e estaduais.

\section{OBJETIVO}

Analisar a variação temporal das neoplasias uterinas na população feminina do estado de Alagoas.

\section{MÉTODO}

Trata-se de um estudo transversal, de caráter descritivo e retrospectivo, com abordagem quantitativa, desenvolvido a partir dos registros documentais referentes ao perfil de morbimortalidade do câncer de colo e corpo uterinos no estado de Alagoas, entre os anos de 2007 e 2016.

A coleta de dados foi desenvolvida nos distintos sistemas de informação disponíveis no Departamento de Informática do SUS, a saber: Sistema de Informações Hospitalares e Sistema de Mortalidade. A amostra foi constituída de todos os casos de câncer uterino diagnosticados no território alagoano, considerando os registros de óbitos e internações hospitalares nos serviços públicos e privados da rede de atenção às neoplasias uterinas do estado de Alagoas.

Utilizaram-se formulários organizacionais, para sistematizar as informações coletadas, contendo as seguintes variáveis: casos diagnosticados, internações hospitalares e óbitos por câncer de útero, faixa etária, microrregião de procedência e localização primária da neoplasia uterina. As informações foram agrupadas em banco de dados, por meio de planilhas eletrônicas, para facilitar a análise dos dados, que foi desenvolvida por meio de estatística descritiva de frequências absoluta $(\mathrm{N})$ e relativa (\%). A fundamentação teórica para a discussão do estudo foi embasada na literatura especializada.

No que tange aos aspectos éticos, a presente pesquisa consolida apenas dados secundários, extraídos de sistema de informação de domínio público, prescindindo da submissão ao Comitê de Ética em Pesquisa, já que se apresenta em consonância com a Resolução No . 466/12 do Conselho Nacional de Saúde - CNS/CONEP.

\section{RESULTADOS}

De 2007 a 2016, segundo o Instituto Nacional 
do Câncer, 3.525 mulheres foram diagnosticadas com neoplasias de útero no estado de Alagoas, com média anual de 352,5 casos novos ( $\pm 64,3)$. De acordo com a Tabela 1, no ano de 2007 houve o maior registro de casos novos de neoplasias uterinas no território alagoano, com
503 diagnósticos (14,3\%). Sequencialmente, observa-se redução significativa $(24,6 \%)$ dos casos diagnosticados no ano de 2007, porém com anuais oscilações numéricas até o ano de 2016, com média de 336 casos novos/ano $( \pm 38,7)$.

Tabela 1. Quantitativo de diagnósticos de neoplasias de útero no estado de Alagoas de 2007 a 2016

\begin{tabular}{lcccccccccc}
\hline & \multicolumn{10}{c}{ Ano do diagnóstico } \\
\cline { 2 - 10 } & $\mathbf{2 0 0 7}$ & $\mathbf{2 0 0 8}$ & $\mathbf{2 0 0 9}$ & $\mathbf{2 0 1 0}$ & $\mathbf{2 0 1 1}$ & $\mathbf{2 0 1 2}$ & $\mathbf{2 0 1 3}$ & $\mathbf{2 0 1 4}$ & $\mathbf{2 0 1 5}$ & $\mathbf{2 0 1 6}$ \\
\hline Número de diagnósticos & 503 & 379 & 296 & 321 & 379 & 387 & 336 & 326 & 322 & 276 \\
Proporção (\%) & 14,3 & 10,7 & 8,4 & 9,1 & 10,8 & 11 & 9,5 & 9,2 & 9,2 & 7,8 \\
\hline
\end{tabular}

Fonte: MS/INCA.

Na Tabela 2, identifica-se a distribuição das internações hospitalares decorrente de neoplasias de útero na população alagoana, segundo as faixas etárias, entre os anos de 2007 e 2016. Assim, é possível inferir que houve 4.540 registros de internações hospitalares nas instituições assistenciais de saúde que compõem a rede de atenção à mulher acometida por neoplasias uterinas, com média de
454 internações/ano $( \pm 77,2)$.

Ademais, é possível identificar que apenas o público feminino de 5 a 14 anos não foi notificado quanto às internações hospitalares por câncer de útero. Por outro lado, há uma maior concentração dos casos em mulheres de 25 a 79 anos, com um total de 4.402 registros hospitalares $(96,96 \%)$.

Tabela 2. Quantitativo de internações por neoplasias de útero no estado de Alagoas, segundo faixas etárias, de 2007 a 2016.

\begin{tabular}{|c|c|c|c|c|c|c|c|c|c|c|c|}
\hline \multirow{2}{*}{ Faixa Etária } & \multicolumn{10}{|c|}{ Ano da internação } & \multirow{2}{*}{ Total } \\
\hline & 2007 & 2008 & 2009 & 2010 & 2011 & 2012 & 2013 & 2014 & 2015 & 2016 & \\
\hline $00-04$ & 0 & 0 & 0 & 0 & 0 & 0 & 0 & 0 & 1 & 1 & 2 \\
\hline $15-19$ & 0 & 0 & 1 & 4 & 2 & 0 & 0 & 2 & 3 & 5 & 17 \\
\hline $20-24$ & 8 & 6 & 11 & 1 & 6 & 0 & 5 & 3 & 4 & 7 & 51 \\
\hline $25-29$ & 26 & 23 & 16 & 16 & 12 & 18 & 11 & 17 & 16 & 10 & 165 \\
\hline $30-34$ & 40 & 42 & 26 & 28 & 43 & 37 & 37 & 27 & 37 & 22 & 339 \\
\hline $35-39$ & 55 & 81 & 57 & 50 & 76 & 85 & 55 & 42 & 44 & 75 & 620 \\
\hline $40-44$ & 54 & 121 & 69 & 72 & 109 & 96 & 85 & 65 & 56 & 74 & 801 \\
\hline $45-49$ & 62 & 97 & 62 & 79 & 113 & 96 & 98 & 56 & 70 & 60 & 793 \\
\hline $50-54$ & 55 & 46 & 44 & 30 & 71 & 34 & 51 & 66 & 52 & 44 & 493 \\
\hline $55-59$ & 58 & 18 & 22 & 18 & 49 & 27 & 29 & 44 & 58 & 38 & 361 \\
\hline $60-64$ & 43 & 9 & 10 & 19 & 25 & 28 & 35 & 42 & 34 & 42 & 287 \\
\hline $65-69$ & 47 & 14 & 3 & 14 & 45 & 18 & 27 & 35 & 21 & 27 & 251 \\
\hline $70-74$ & 23 & 8 & 3 & 11 & 32 & 18 & 21 & 12 & 26 & 22 & 176 \\
\hline $75-79$ & 14 & 11 & 4 & 4 & 16 & 10 & 13 & 17 & 8 & 19 & 116 \\
\hline 80 ou mais & 18 & 3 & 2 & 4 & 8 & 1 & 6 & 6 & 16 & 4 & 68 \\
\hline Total & 503 & 479 & 330 & 350 & 607 & 468 & 473 & 434 & 446 & 450 & 4.540 \\
\hline
\end{tabular}

Fonte: MS/DATASUS/SIH.

Nos dez anos analisados, constata-se que houve redução das internações hospitalares por câncer de útero na maioria das faixas etárias descritas. Entretanto, destacase o expressivo quantitativo de mulheres acometidas em faixas etárias prioritárias do Programa de Rastreamento do Câncer do Colo do Útero vigente (25 e 64 anos), bem como é notória e relevante a expressão na população com idade de 65 anos ou mais, registrando 611 ocorrências, o que equivale a $13,46 \%$ das internações hospitalares.

O quantitativo de óbitos decorrentes de neoplasias uterinas, como causas primárias, na população alagoana encontra-se descrito na Tabela 3. No período em análise, 
Misael JR, et al. Panorama das neoplasias uterinas no período de 2007 a 2016 no estado de Alagoas - Brasil.

foram notificados 1.391 óbitos de mulheres por neoplasias de útero, com média de 139 óbitos anuais $( \pm 13,99)$. Vale ressaltar que há uma tendência progressiva ao longo dos últimos anos, cuja maior incidência de óbitos foi registrada no ano de 2014, com um total de 156 mortes.

Quanto à localização primária do acometimento uterino, nota-se que há maior número de óbitos por neoplasias do colo uterino, com equivalência de 64,63\% das neoplasias uterinas (899 óbitos) e elevação quantitativa no último triênio analisado. Contribuindo com o presente panorama de mortalidade no estado alagoano, as neoplasias do corpo uterino e de porções não especificadas apresentaram conjuntamente um total de 492 óbitos.

Considerando a mortalidade da população alagoana nos diversos centros de referências estaduais e nacionais, a Tabela 4 demonstra o quantitativo de óbitos por neoplasias uterinas, segundo a faixa etária, nos anos de 2007 a 2016.

Tabela 3. Quantitativo de óbitos por neoplasias de útero no estado de Alagoas, segundo localização neoplásica primária, de 2007 a 2016

\begin{tabular}{|c|c|c|c|c|c|c|c|c|c|c|c|}
\hline \multirow{2}{*}{ Localização da Neoplasia } & \multicolumn{10}{|c|}{ Ano do Óbito } & \multirow{2}{*}{ Total } \\
\hline & 2007 & 2008 & 2009 & 2010 & 2011 & 2012 & 2013 & 2014 & 2015 & 2016 & \\
\hline Colo uterino & 73 & 95 & 82 & 85 & 90 & 94 & 84 & 99 & 103 & 94 & 899 \\
\hline Corpo uterino & 6 & 10 & 4 & 13 & 12 & 9 & 9 & 15 & 16 & 18 & 112 \\
\hline Útero - porção não especificada & 34 & 44 & 39 & 31 & 39 & 42 & 38 & 42 & 34 & 37 & 380 \\
\hline Total & 113 & 149 & 125 & 129 & 141 & 145 & 131 & 156 & 153 & 149 & 1.391 \\
\hline
\end{tabular}

Fonte: MS/DATASUS/SIM

Tabela 4. Quantitativo de óbitos por neoplasias de útero no estado de Alagoas, segundo a faixa etária, de 2007 a 2016

\begin{tabular}{lcccccccc}
\hline & \multicolumn{10}{c}{ Faixa Etária } \\
\cline { 2 - 9 } & $\begin{array}{c}\mathbf{1 5} \text { a 19 } \\
\text { anos }\end{array}$ & $\begin{array}{c}\mathbf{2 0} \text { a 29 } \\
\text { anos }\end{array}$ & $\begin{array}{c}\mathbf{3 0} \text { a 39 } \\
\text { anos }\end{array}$ & $\begin{array}{c}\mathbf{4 0} \text { a 49 } \\
\text { anos }\end{array}$ & $\begin{array}{c}\mathbf{5 0} \text { a 59 } \\
\text { anos }\end{array}$ & $\begin{array}{c}\mathbf{6 0} \text { a 69 } \\
\text { anos }\end{array}$ & $\begin{array}{c}\mathbf{7 0} \text { a 79 } \\
\text { anos }\end{array}$ & $\begin{array}{c}\mathbf{8 0} \text { anos e } \\
\text { mais }\end{array}$ \\
\hline $\begin{array}{l}\text { Número de } \\
\text { Óbitos }\end{array}$ & 2 & 37 & 182 & 311 & 292 & 262 & 196 & 136 \\
Proporção (\%) & 0,14 & 2,61 & 12,84 & 21,93 & 20,59 & 18,48 & 13,82 & 9,59 \\
\hline
\end{tabular}

Fonte: MS/DATASUS/SIM.

Nesse contexto, a partir de 15 anos, todas as faixas etárias apresentaram mortalidade específica, com destaque para a população adulta, que dispõe de um total de 822 óbitos $(57,97 \%)$, especialmente em mulheres com idade a partir da quarta década de vida. Ratifica-se ainda a elevada prevalência no público idoso, com um total de 594 óbitos, ou seja, $41,89 \%$ da mortalidade por câncer uterino.
A análise do quantitativo de óbitos por neoplasias uterinas a partir do Plano Diretor de Regionalização do estado de Alagoas indica a dinâmica epidemiológica regional e, por meio das ocorrências em cada microrregião específica, impacta diretamente nos aspectos gerenciais das ações e serviços vinculados às políticas públicas de saúde da mulher.

Tabela 5. Quantitativo de óbitos por neoplasias de útero no estado de Alagoas, segundo a microrregião de saúde de Alagoas, de 2007 a 2016

\begin{tabular}{lcccccccccc}
\hline & \multicolumn{10}{c}{ Microrregião de Saúde } \\
\cline { 2 - 10 } & $\mathbf{1}^{\mathbf{a}}$ & $\mathbf{2}^{\mathbf{a}}$ & $\mathbf{3}^{\mathbf{a}}$ & $\mathbf{4}^{\mathbf{a}}$ & $\mathbf{5}^{\mathbf{a}}$ & $\mathbf{6}^{\mathbf{a}}$ & $\mathbf{7}^{\mathbf{a}}$ & $\mathbf{8}^{\mathbf{a}}$ & $\mathbf{9}^{\mathbf{a}}$ & $\mathbf{1 0}^{\mathbf{a}}$ \\
\hline Número de Óbitos & 664 & 58 & 103 & 69 & 93 & 80 & 166 & 53 & 69 & 63 \\
Proporção & 46,8 & 4,09 & 7,27 & 4,87 & 6,56 & 5,64 & 11,71 & 3,75 & 4,87 & 4,44 \\
\hline
\end{tabular}

Fonte: MS/DATASUS/SIM. 
A Tabela 5 descreve as frequências absolutas e relativas dos óbitos femininos secundários às neoplasias uterinas em função da microrregião de saúde de Alagoas. Isto posto, as microrregiões de saúde que apresentam maior número de usuárias correspondem a áreas territoriais adscritas com os maiores contingentes populacionais do Estado, com os municípios-polos de Maceió ( $1^{\mathrm{a}}$ região) e Arapiraca ( $7^{\mathrm{a}}$ região), que juntos concentram 58,51\% dos óbitos locais (830 registros).

\section{DISCUSSÃO}

O câncer uterino é um dos que apresenta maior potencial de prevenção e cura, em especial o do colo do útero, devido a sua lenta evolução, passando por fases detectáveis e curáveis. Porém falhas no desempenho dos programas de rastreamento voltados para a saúde da mulher prejudicam o alcance de metas proposta ao longo dos anos. Não obstante dos resultados encontrados no presente estudo, alguns pesquisadores analisaram a distribuição espacial dos indicadores pactuados para o câncer do colo do útero no Brasil e apontaram que, apesar de estruturados, os dados de morbimortalidade ainda se encontram acima das metas pactuadas em algumas regiões brasileiras, como o eixo sul-sudeste ${ }^{3}$.

Conforme Tabela 1, o ano de 2007 apresentou o maior número de casos diagnosticados de neoplasias uterinas no território alagoano na última década. Este fator pode ser reflexo da eficiente implantação de políticas de saúde femininas, como por exemplo, a Política Nacional de Atenção Integral à Saúde da Mulher, lançada em 2004 pelo Ministério da Saúde, que incluiu o plano de ação de 2004-2007, tendo como um dos objetivos reduzir a morbimortalidade por câncer na população feminina no Brasil, especialmente por causas evitáveis em todos os ciclos de vida e nos diversos grupos populacionais.

Tal intervenção apresentou como meta prioritária o aumento, em no mínimo $30 \%$, da realização do exame de Papanicolau na população de risco à época (35 a 49 anos). Além disso, as estratégias foram organizadas em função da territorialização, viabilizando a descentralização em municípios-polos, consolidando a oferta da rede de referência e contrarreferência para diagnóstico e tratamento de câncer de colo uterino, identificando as diferenças regionais e as desigualdades de acesso das ações preventivas $^{8}$.

Em função do tratamento específico, entre os atributos dos serviços que compõem a Atenção Primária em Saúde, cabe aos profissionais encaminhar imediatamente para os serviços especializados, quando da suspeição ou confirmação diagnóstica. No que compete às instituições de referência no estado de Alagoas para o câncer de útero, a Tabela 2 demonstra elevados registros anuais de internações hospitalares, embora com tendência regressiva ao longo dos anos.
Segundo a Organização Mundial de Saúde, a incidência desse câncer aumenta nas mulheres entre 30 e 39 anos, e atinge seu pico na quinta ou sexta década de vida. Antes dos 25 anos de idade, prevalecem as infecções por HPV e as lesões de baixo grau, que regredirão espontaneamente, na maioria dos casos. Por outro lado, na população idosa, acima de 65 anos, o risco de desenvolvimento do câncer cervical é reduzido, dada à sua lenta evolução ${ }^{9}$, embora os achados da Tabela 2 apresentem eminente quantitativo de mulheres diagnosticadas com câncer uterino na população alagoana acima de 60 anos de idade - destacando a necessidade de amplificação das faixas etárias prioritárias para os pertinentes rastreamentos diagnósticos.

Para tal, os sistemas de informação em saúde devem permitir que todos os envolvidos nas ações dispostas na Atenção Primária à Saúde tenham contínuo acesso a relatórios informatizados, para que se possa retroalimentar o planejamento dos diferentes programas assistenciais. Ainda, faz-se necessário o aprimoramento dos sistemas de contrarreferência, já que as usuárias referidas aos serviços da alta complexidade ficam desprovidas do seguimento na Atenção Primária à Saúde, na maioria dos casos, mesmo demandando cuidados compartilhados durante seu processo de reabilitação ${ }^{10}$.

Ao analisar a Tabela 3, observa-se discreta elevação quanto ao número de óbitos por neoplasias de colo uterino, entre os anos de 2014 e 2016. Entre os cânceres, as neoplasias de colo uterino há maior impacto para a saúde pública, em detrimento da grande oferta e acessibilidade nos serviços vinculados ao Sistema Único de Saúde, bem como pela possibilidade de prevenção, rastreamento e cura $^{10}$. O estado de Alagoas segue a tendência da região nordeste brasileira na estabilização do número de casos, sendo atualmente essa neoplasia a terceira causa de localização primária em incidência e mortalidade por câncer em mulheres no país, excluindo o câncer de pele não melanoma ${ }^{11}$.

Quanto à idade de maior ocorrência de câncer uterino na população alagoana feminina, as faixas etárias que apresentaram maior número de registros de óbitos correspondem aos 25 a 60 anos, embora as adolescentes apresentem variáveis índices de vulnerabilidade para este agravo, uma vez que se identifica atualmente o precoce início da vida sexual, além da multiplicidade de parceiros, que as aproximam do maior risco de exposição a agentes infecciosos, culminando com interferências nas esferas reprodutiva e sexual ${ }^{12}$.

Estudos revelam que o Papiloma Vírus Humano (HPV), é o principal agente oncogênico do câncer de colo uterino, sendo que o contágio usualmente ocorre no início da vida sexual, principalmente na adolescência, e decorre também do maior número de parceiros sexuais ${ }^{13}$. A incidência do HPV em adolescentes foi ilustrada em pesquisas, revelando taxas de até $48,9 \%$ em grupos 
amostrais, das quais cerca de $28,5 \%$ apresentaram infecções múltiplas, sendo o HPV-16 o subtipo mais comum ${ }^{14}$.

Entretanto, o Ministério da Saúde destaca que a infecção em mulheres com menos de 30 anos regrida espontaneamente, com rara evolução para o câncer de colo uterino ${ }^{11}$. Tais evidências destacam a importância da captação precoce de mulheres com história sexual inicializada, conforme as Diretrizes para o Rastreamento do Câncer do Colo do Útero ${ }^{6}$, priorizando o público de 25 a 64 anos para o devido rastreamento com o exame de Papanicolaou ${ }^{15}$.

É consenso que o rastreamento organizado e amplificado deve incluir a comunicação e o processo contínuo de educação para a saúde como mecanismos de prevenção primária ${ }^{11}$, incluindo principalmente a utilização de métodos de barreiras. Ademais, um fator altamente eficaz na prevenção relatado na literatura consiste na vacinação contra o HPV, que está disponível na rede pública de saúde para meninas de 9 a 13 anos, prevenindo contra quatro subtipos do vírus, apesar de deixar grande parcela da população feminina sem cobertura vacinal, já que mesmo as mulheres sexualmente ativas, e possíveis portadoras de HPV, poderiam ser imunizadas contra os subtipos que não tiveram exposição ${ }^{16}$, mesmo não eliminando a necessidade de prevenção secundária por meio do rastreamento.

Em consonância com a Tabela 5, em função do território administrativo, é possível conferir que os maiores contingentes populacionais estão concentrados nas microrregiões que demonstram elevados números de óbitos por câncer de útero. Como exemplo, a $1^{\mathrm{a}}$ microrregião de saúde, que apresenta a capital alagoana como municípiopolo, congrega $46,8 \%$ dos óbitos registrados em todo o estado. Tal fato deve estar relacionado com a baixa cobertura da Estratégia de Saúde da Família no município de Maceió, com adscrição inferior a $30 \%{ }^{17}$, definindo à dificuldade de acesso aos serviços de saúde locais.

Sabe-se que há consenso na determinação da prevalência de câncer uterino em função da limitada acessibilidade $^{18}$, mas a identificação da distribuição territorial em Alagoas, com base no Plano Diretor de
Regionalização, favorece o adequado planejamento das Políticas de Saúde da Mulher, com o envolvimento de ações e serviços que integrem uma rede regionalizada e hierarquizada e constituem um sistema especializado de combate ao câncer de útero, alocando recursos humanos qualificados e tecnologias específicas para os distintos níveis de prevenção ${ }^{19}$.

\section{CONCLUSÃO}

Através de recorte temporal, o presente estudo revela o comportamento dos casos de câncer uterino na população feminina alagoana nos últimos dez anos, sobretudo destacando os elevados índices de hospitalizações e óbitos no Estado. Apesar dos esforços governamentais para minimizar o perfil epidemiológico, é notória a fragilidade nas ações de saúde da mulher em todo o território de Alagoas, principalmente no que tange às demandas de inclusão para as prevenções primárias e secundária.

Ainda, apesar da concentração dos registros relacionados às neoplasias uterinas no público adulto, faz necessário o efetivo planejamento e implementação das políticas públicas de saúde da mulher, especialmente com a capacidade extensiva às populações pertencentes à chamada terceira idade, já que o público idoso feminino contribui para a manutenção do panorama evidenciado ao longo dos últimos anos.

Reconhece-se que analisar detalhadamente a gênese das variáveis vinculadas às neoplasias uterinas requer estudos aprofundados sobre os levantamentos apontados ao longo do presente estudo. Entretanto, os dados coletados e analisados podem estimular gerentes dos distintos programas transversais de saúde da mulher no Estado de Alagoas a analisar a marcante transição epidemiológica do câncer uterino e definir pactuações que viabilizem os princípios e diretrizes das políticas públicas direcionadas ao público feminino, sobretudo na amplificação de ações e serviços de saúde com capacidade preventiva, refletindo positivamente na saúde e qualidade de vida desse público específico.

Contribuição dos autores: Jailton Rocha Misael: Participou do delineamento do estudo, coleta de dados, análise e escrita dos resultados. Claudio José dos Santos Júnior: Participou da escrita do artigo, da análise e discussão dos dados e da revisão final do artigo. Marcicléa Macedo de Lima Batista: Participou da coleta e tabulação dos dados. Ilma Falcão Silva: Participou da escrita dos resultados, interpretação e geração de tabelas e indicadores. Lídia Nathaly Gomes dos Santos: Participou da escrita dos resultados e discussão. Fernando Batista Chicuta da Rocha: Participou da escrita da discussão. Amanda Maria Monteiro Ferreira: Participou do delineamento, coordenação e orientação do estudo, análise, interpretação e discussão dos dados e da revisão final do artigo.

\section{REFERÊNCIAS}

1. Souza MFM, Malta DC, França EB, Barreto ML. Transição da saúde e da doença no Brasil e nas Unidades Federadas durante os 30 anos do Sistema Único de Saúde. Cienc Saude Coletiva. 2018;23(6):1737-50. http://dx.doi.org/10.1590/141381232018236.04822018 .

2. Bray F, Jemal A, Grey N, Ferlay J, Forman D. Global cancer transitions according to the Human Development Index (2008-2030): a population based study. Lancet Oncol. 2012;13(8):790-801. https://doi.org/10.1016/S14702045(12)70211-5.

3. GirianelliI VR, GamarraII CJ, SilvaIII GA. Os grandes contrastes na mortalidade por câncer do colo uterino e de mama no Brasil. Rev Saúde Pública. 2014;48(3):459-67. http://dx.doi.org/10.1590/S0034-8910.2014048005214. 
4. World Health Organization. ICO Information Centre on HPV and Cervical Cancer (HPV Information Centre). Summary report on HPV and cervical cancer statistics in Brazil. 2014. Avaliable from: http://www.hpvcentre.net.

5. Instituto Nacional de Câncer. Coordenação de Prevenção e Vigilância. Estimativa 2018: incidência de câncer no Brasil. 2018. Disponível em: https://www.inca.gov.br/ estimativa/2018/estimativa-2018.pdf

6. Instituto Nacional de Câncer José Alencar Gomes da Silva. Coordenação de Prevenção e Vigilância. Divisão de Detecção Precoce e Apoio à Organização de Rede. Diretrizes brasileiras para o rastreamento do câncer do colo do útero. 2016. Disponível em: http://www2.inca.gov.br/ wps/wcm/connect/9000f2004b39c00db985bf66c974cd7f/ Diretrizes + Brasileiras +2016 _vers $\% \mathrm{C} 3 \% \mathrm{~A} 3 \mathrm{o}+$ Consulta $+\mathrm{P}$ $\% \mathrm{C} 3 \%$ BAblica.pdf.

7. Olhê L, Oliveira RC, Campanelli RF, Nogueira LDP. Papanicolau na terceira idade: um desafio para a enfermagem. Rev Fabibe. 2013:78-86. Disponível em: http://www. unifafibe.com.br/revistasonline/arquivos/revistafafibeonline/ sumario/28/11122013190008.pdf.

8. Ribeiro CM, Silva GA. Avaliação da produção de procedimentos da linha de cuidado do câncer do colo do útero no Sistema Único de Saúde do Brasil em 2015. Epidemiol Serv Saúde. 2018;27(1):e20172124. http://dx.doi. org/10.5123/s1679-49742018000100004.

9. Instituto Nacional de Câncer. Ações de Controle do Câncer do Colo do Útero. Gestor e Profissional da Saúde. Detecção Precoce no Brasil. 2018. Disponível em: https://www.inca. gov.br/en/node/1194.

10. Brasil. Ministério da Saúde. Cadernos de Atenção Básica 13. Controle dos cânceres de colo do útero e da mama. Brasília; 2013. p.18. Disponível em: http://bvsms.saude.gov.br/bvs/ publicacoes/controle_canceres_colo_utero_2013.pdf.

11. Instituto Nacional de Câncer. Ações de controle do câncer do colo do útero. 2018. Disponível em: https://www.inca.gov.br/ controle-do-cancer-do-colo-do-utero/conceito-e-magnitude.

12. Melo WA, Pelloso SM, Alvarenga A, Carvalho MDB. Fatores associados a alterações do exame citopatológico cérvico-uterino no Sul do Brasil. Rev Bras Saude Mater Infant. 2017;17(4):637-43. http://dx.doi.org/10.1590/180693042017000400002 .

13. Abreu MNS, Soares AD, Ramos DAO, Soares FV, Nunes FG, Valadão AF, et al. Conhecimento e percepção sobre o HPV na população com mais de 18 anos da cidade de Ipatinga, MG, Brasil. Ciênc Saúde Coletiva. 2018;23(3):849-60. http:// dx.doi.org/10.1590/1413-81232018233.00102016.

14. Del Río-Ospina L, Soto-De León SC, Camargo M, Sánchez $\mathrm{R}$, Mancilla CL, Patarroyo ME, et al. The prevalence of highrisk HPV types and factors determining infection in female colombian adolescents. PLoS One. 2016;11(11):e0166502. https://doi.org/10.1371/journal.pone.0166502.

15. Brasil. Ministério da Saúde. Secretaria de Atenção à Saúde. Departamento de Atenção Básica. Controle dos cânceres do colo do útero e da mama. 2a ed. Brasília (DF): Editora do Ministério da Saúde; 2013.

16. Sanches TT, Siqueira-Oliveira T, Papp-Moretti C, TovaniPalone MR, Hishinuma G. Evolução do sistema público de saúde no Brasil frente ao estágio atual da prevenção do câncer de colo uterino em mulheres jovens e adolescentes. Rev Fac Med. 2017;65(1):115-20. http://dx.doi.org/10.15446/ revfacmed.v65n1.56855.

17. Silva MAP, Menezes RCE, Oliveira MAA, Longo-Silva G, Asakura L. Atenção Básica em Alagoas: expansão da Estratégia Saúde da Família, do NASF e do componente alimentação/nutrição. Saúde Debate. 2014;38(103):720-32. http://dx.doi.org/10.5935/0103-1104.20140066.

18. McDonald TJ, Goldberg DW, Scarinci IC, Castle PE, Cuzick J, Robertson M, Wheeler CM. Health service accessibility and risk in cervical cancer prevention: comparing rural versus nonrural residence in New Mexico. J Rural Health. 2017;33:382-92. https://doi.org/10.1111/jrh.12202.

19. Santos, L. Região de saúde e suas redes de atenção: modelo organizativo-sistêmico do SUS. Ciênc Saúde Coletiva. 2017;22(4):1281-9. https://doi.org/10.1590/141381232017224.26392016

Recebido: 23.03 .19

Aceito: 15.07 .19 